\title{
Diabetes, aging, and their tissue complications
}

Rick Bucala

Yale University School of Medicine, New Haven, Connecticut, USA.

\begin{abstract}
The inactivation of NO by advanced glycation endproducts (AGEs), which accumulate on tissue proteins as a function of age and hyperglycemia, focused attention on the role of these ubiquitous posttranslational modifications in acquired impairments of vascular reactivity and other signaling processes. This observation occurred during a watershed period of basic and translational research in glycation that encompassed new pathologic phenomena and novel intervention strategies. How has the AGE paradigm for the tissue complications of aging and diabetes fared since the identification of the link between these glycation products and NO inactivation, and what lessons may be offered for future investigations?
\end{abstract}

\section{A serendipitous discovery}

The discovery of NO as the active constituent of endothelium-derived relaxing factor prompted new lines of investigations into cardiopulmonary physiology, innate immunity, neuroscience, and metabolism (1). The 1991 report published by my team in the JCI that revealed protein glycation adducts, which accumulate with age on long-lived proteins and in greater amounts in diabetes, react with and inactivate $\mathrm{NO}$ developed serendipitously (2). During my internal medicine training, Eugene Braunwald taught residents (at the bedside) the variants of anginal syndromes, including those mediated not by luminal obstruction but by an imbalance in vasoactive factors. Following residency, I conducted research with Anthony Cerami in glycation biochemistry that set the stage for considering how the interposition of glycation adducts, which result from the rearrangement of the glucose-derived Amadori reaction on longlived proteins, could functionally impede NO-mediated vasodilatation. Glycationmediated protein crosslinking was thought to account for senile cataracts and for ageand diabetes-related decreases in vascular wall compliance and glomerular basement membrane function, and ultimately, it was hypothesized to contribute to a host of age-related phenomena, including systolic hypertension, diastolic cardiac dysfunction, and the inexorable decreases in tissue compliance that compromise pulmonary or musculoskeletal function. The ability of these advanced glycation endproducts

Conflict of interest: The author has declared that no conflict of interest exists.

Citation for this article: J Clin Invest. 2014; 124(5):1887-1888. doi:10.1172/JCI75224.
(AGEs) to covalently crosslink connective tissue matrix proteins could be viewed as but one adverse property of these chemical modifications, and the AGE-dependent impairment of NO-dependent vasodilation and cellular signaling broadened the pathologic impact of glycation chemistry $(2,3)$.

\section{Targeting AGE-mediated damage}

Views of the biologic consequences of glucose-related macromolecular damage expanded substantially in the ensuing years to encompass genotoxic DNA damage (4), aminolipid glycation and oxidation (5), amyloid deposition (6), and intracellular glycation and redox stress (7). The molecules participating in glycation damage also expanded beyond endogenous reducing sugars and their metabolites to include tobacco smoke (8) and diet (9), with each observation lending further support to an overarching AGE hypothesis for the sequelae of aging and the long-term cardiovascular complications of diabetes and for renal insufficiency, which impairs clearance of reactive AGEs. Earnest attempts were made to reduce AGE-mediated tissue damage pharmacologically. These efforts were predicated on tractable points in the biochemical pathways considered responsible for reactive dicarbonyl formation, conjugated double bond formation, and covalent crosslinking.

How have these efforts fared, and what are the realistic prospects for such targeted interventions? Beyond the global diabetes epidemic, the number of Americans over age 65 is estimated to reach $19 \%$ of the population by 2030 , providing an imperative to better understand the pathophysiology of aging and to devise new therapies. Initial pharmacologic efforts to reduce
AGE-associated damage were directed at inactivating glucose-derived, $\alpha$-dicarbonylreactive intermediates with a small competing nucleophile in order to interrupt the chemical progression to crosslinking (10). Despite auspicious preclinical data, two large double-blind randomized clinical trials in diabetic nephropathy (ACTION I, II) did not achieve primary end points. More audacious was the concept of a pharmacologic "AGE breaker" or agent to chemically attack, destabilize, and cleave AGE crosslinks and reverse established age- or diabetes-related tissue damage (11). These thiazolium-based pharmacophores improved cardiovascular function in animal models, and enhancements in vasodilation and diastolic function were reported in small, uncontrolled human studies; however, data from randomized clinical trials were not encouraging (12). Other approaches to reduce AGE-associated effects are continuing, including restricting the consumption of AGE-containing foods and testing the effects of $B$ vitamins or their derivatives (13), which offer the broader action of interfering with intracellular glycation pathways and cellular damage. It remains to be seen whether medically significant effects will result from these interventions, either alone or in combination with modalities that reduce glycemia, the main driver of AGE formation.

\section{What lies ahead?}

Despite the conceptual promise, why have AGE-directed therapies been disappointing? The financial exigencies of drug development often force clinical testing of the first, but not necessarily the "best," drug candidate. Better knowledge of the targeted chemical moieties and in vivo pathways for AGE formation and removal is critical and will necessarily encompass critical information about competing pathways for product progression or degradation, which may involve oxidative stress as well as cellular pathways of AGE recognition and matrix remodeling. It is gratifying that recent developments in glycation chemistry predict actual NO quenching species (14) and that functional vasospasm may be more effectively treated by modalities 
such as calcium channel blockers. Despite the availability of quantitative immunoassays and more sensitive and robust liquid chromatography mass spectrometry methodologies, straightforward methods to monitor important AGE intermediates and their progression to macromolecular damage are lacking, especially in living biologic systems. Information about tissue damage remains very dependent on in vitro and in vivo model studies, but even the study of experimental disease may be limited due to evidence that the tissue damage and crosslinking signatures in experimental animals differ from those in long-lived humans (15). Given the explosion of information in medical genetics and genetic modifiers of disease, it could be useful to investigate more closely whether certain individuals are predisposed to rapid or slow progression of AGE damage and whether pharmacologic intervention confers greater benefit in a particular subgroup of individuals. That the many sequelae of aging may be unified in their pathogenesis by glycation, nevertheless, remains highly attractive conceptually and deserves the convergence of continued multidisciplinary investigations. Much remains to be done.

\section{Acknowledgments}

The author expresses gratitude to David Spiegel for helpful discussions and to the Brookdale Foundation for their support of aging research.

Address correspondence to: Rick Bucala, Professor of Medicine, Yale University School of Medicine, TAC S521, PO Box 208031, 300 Cedar Street, New Haven, Connecticut 06520-8031, USA. Phone: 203.737.1453; Fax: 203.785.7053; E-mail: Richard.Bucala@Yale.edu.

1. Ignarro LJ, Buga GM, Wood KS, Byrns RE, Chaudhuri G. Endothelium-derived relaxing factor produced and released from artery and vein is nitric oxide. Proc Natl Acad Sci U S A. 1987; 84(24):9265-9269.

2. Bucala R, Tracey KJ, Cerami A. Advanced glycosylation products quench nitric oxide and mediate defective endothelium-dependent vasodilatation in experimental diabetes. J Clin Invest. 1991;87(2):432-438.

3. Hogan M, Cerami A, Bucala R. Advanced glycosylation endproducts block the antiproliferative effect of nitric oxide. Role in the vascular and renal complications of diabetes mellitus. J Clin Invest. 1992; 90(3):1110-1115

4. Lee AT, Plump A, DeSimone C, Cerami A, Bucala R. A role for DNA mutations in diabetes-associated teratogenesis in transgenic embryos. Diabetes. 1995;44(1):20-24.

5. Bucala R, Makita Z, Koschinsky T, Cerami A, Vlassara H. Lipid advanced glycosylation: pathway for lipid oxidation in vivo. Proc Natl Acad Sci U S A. 1993;90(14):6434-6438.

6. Smith MA, et al. Advanced Maillard reaction end products are associated with Alzheimer disease pathology. Proc Natl Acad Sci U S A. 1994; 91(12):5710-5714.

7. Brownlee M. Biochemistry and molecular cell biology of diabetic complications. Nature. 2001; 414(6865):813-820.

8. Cerami C, et al. Tobacco smoke is a source of toxic reactive glycation products. Proc Natl Acad Sci US A. 1997;94(25):13915-13920.

9. Koschinsky T, et al. Orally absorbed reactive glycation products (glycotoxins): an environmental risk factor in diabetic nephropathy. Proc Natl Acad Sci US A. 1997;94(12):6474-6479.

10. Brownlee M, Vlassara H, Kooney A, Ulrich P, Cerami A. Aminoguanidine prevents diabetes-induced arterial wall protein cross-linking. Science. 1986; 232(4758):1629-1632.

11. Vasan $S$, et al. An agent cleaving glucose-derived protein crosslinks in vitro and in vivo. Nature. 1996; 382(6588):275-278.

12. Engelen L, Stehouwer CD, Schalkwijk CG. Current therapeutic interventions in the glycation pathway: evidence from clinical studies. Diabetes Obes Metab. 2013;15(8):677-689.

13. Hammes HP, et al. Benfotiamine blocks three major pathways of hyperglycemic damage and prevents experimental diabetic retinopathy. Nat Med. 2003; 9(3):294-299.

14. Wang T, Kartika R, Spiegel DA. Exploring posttranslational arginine modification using chemically synthesized methylglyoxal hydroimidazolones. J Am Chem Soc. 2012;134(21):8958-8967.

15. Sell DR, Monnier VM. Molecular basis of arterial stiffening: role of glycation - a mini-review. Gerontology. 2012;58(3):227-237. 